Association for Information Systems AIS Electronic Library (AISeL)

Wirtschaftsinformatik Proceedings 2005

Wirtschaftsinformatik

February 2005

\title{
Steuerungsmodell zur kundenorientierten Entwicklung von E-Government-Dienstleistungen
}

Wolf-Gideon Bleek

Universität Hamburg

Ralf Klischewski

German University in Cairo

Heike Stach

Bundesministerium des Innern

Follow this and additional works at: http://aisel.aisnet.org/wi2005

\section{Recommended Citation}

Bleek, Wolf-Gideon; Klischewski, Ralf; and Stach, Heike, "Steuerungsmodell zur kundenorientierten Entwicklung von EGovernment-Dienstleistungen" (2005). Wirtschaftsinformatik Proceedings 2005. 37.

http://aisel.aisnet.org/wi2005/37

This material is brought to you by the Wirtschaftsinformatik at AIS Electronic Library (AISeL). It has been accepted for inclusion in Wirtschaftsinformatik Proceedings 2005 by an authorized administrator of AIS Electronic Library (AISeL). For more information, please contact elibrary@aisnet.org. 
In: Ferstl, Otto K, u.a. (Hg) 2005. Wirtschaftsinformatik 2005: eEconomy, eGovernment, eSociety; 7. Internationale Tagung Wirtschaftsinformatik 2005. Heidelberg: Physica-Verlag

ISBN: 3-7908-1574-8

(C) Physica-Verlag Heidelberg 2005 


\title{
Steuerungsmodell zur kundenorientierten Entwicklung von E-Government- Dienstleistungen
}

\author{
Wolf-Gideon Bleek \\ Universität Hamburg \\ Ralf Klischewski \\ German University in Cairo \\ Heike Stach \\ Bundesministerium des Innern
}

Zusammenfassung: Damit Investitionen in Online-Angebote der Verwaltung zeitnah zu den erwarteten Qualitätsverbesserungen und Einsparungseffekten führen, richtet sich der Fokus bei der E-Government-Entwicklung zunehmend auf die Abnehmer der Dienstleistungen und deren Anliegen, z. B. von Bürgerinnen und Bürger oder Unternehmen im jeweiligen Zuständigkeitsbereich. Um diese Herangehensweise zu unterstützen, skizziert der vorliegende Beitrag ein neues Steuerungsmodell zur kundenorientierten Entwicklung von E-Government-Dienstleistungen, das zentral auf der Auswertung von Anwenderfeedback beruht. Dieses Modell basiert auf den Erkenntnissen und Erfahrungen der anwendungsorientierten Entwicklung von Web-Anwendungen, die sich aus der jahrzehntelangen Forschung und Praxis der partizipativen Softwareentwicklung ableiten. Darüber hinaus werden die besonderen Bedingungen von E-Government-Projekten (wie z. B. BundOnline 2005) berücksichtigt, um zu einer den Möglichkeiten der Verwaltung gerechten Verfahren und Steuerungsmöglichkeiten zu gelangen.

Schlüsselworte: E-Government, Dienstleistungsentwicklung, Kundenorientierung, Neues Steuerungsmodell, Partizipation, Softwareentwicklung, Entwicklungsprozess, evolutionär, zyklisch

\section{Einleitung}

Nachdem E-Government-Angebote im World Wide Web mittlerweile als Selbstverständlichkeit gelten, tritt die Entwicklung dieser Dienstleistungen in eine neue Phase: der Fokus richtet sich zunehmend auf die Abnehmer der Dienstleistungen 
und deren Anliegen, z. B. von Bürgerinnen und Bürger oder Unternehmen im jeweiligen Zuständigkeitsbereich. Mit Hilfe des Schlagworts „Kundenorientierung“, das schon seit einigen Jahren auch das Verwaltungsdenken beflügelt, soll sicher gestellt werden, dass Investitionen in Online-Angebote zeitnah zu den erwarteten Qualitätsverbesserungen und Einsparungseffekten führen. Gesucht sind Herangehensweisen und Verfahren, die bei Entwicklung von E-Government-Dienstleistungen systematisch und nachhaltig die Anliegen der potenziellen Nutzer einbeziehen und somit zu gesicherter Akzeptanz der Online-Angebote und zu in ihrer KostenNutzen-Relation erfolgreichen E-Government-Projekten führen.

Vor diesem Hintergrund skizziert der vorliegende Beitrag ein neues Steuerungsmodell zur kundenorientierten Entwicklung von E-Government-Dienstleistungen. Es basiert zum einen auf den Erkenntnissen und Erfahrungen der anwendungsorientierten Entwicklung von Web-Anwendungen, die sich aus der jahrzehntelangen Forschung und Praxis der partizipativen Softwareentwicklung ableiten. Zum anderen werden die besonderen Bedingungen von E-Government-Projekten berücksichtigt, um zu einer den Möglichkeiten der Verwaltung gerechten Verfahren und Steuerungsmöglichkeiten zu gelangen. Mit dem bewussten Rekurs auf die Konzepte des New Public Management soll dabei die Perspektive der beteiligten Akteure in den Vordergrund gestellt werden. Gleichzeitig ist damit die These verbunden, dass eine Orientierung am „neuen Steuerungsmodell“ auch die Professionalisierung der Entwicklung von E-Government-Dienstleistungen befördert.

Im nachfolgenden Abschnitt wird am Beispiel der Initiative BundOnline 2005 diskutiert, worin der Handlungsbedarf bei der Dienstleistungsentwicklung besteht. Im dritten Abschnitt wird die kundenorientierte Entwicklung von Web-Anwendungen aus softwaretechnischer Perspektive hergeleitet und deren Herangehensweise im Hinblick auf die Übertragung in die Verwaltung diskutiert. Der vierte Abschnitt skizziert dann das neue Steuerungsmodell für E-Government-Projekte, welches die bisherige Dienstleistungsentwicklung mit ein Bündel von ,kundenorientierten“ Maßnahmen zyklisch integriert und auf der Ebene des Projektmanagements eine professionelle und kooperative Steuerung etabliert.

\section{E-Government ohne Kundenbindung?}

Das Kundenverhältnis im E-Government ist signifikant verschieden von dem allgemein bei Behörden zugrunde gelegten Verhältnis zum Abnehmer ihrer Leistungen: es ist labiler und bedarf einer aktiven Pflege. Erstens ist der elektronische Kanal ein zusätzliches Angebot, von dem bei Nichtgefallen jederzeit auf einen anderen Kanal gewechselt werden kann. Während die Kunden an Service-Verbesserung interessiert sind, streben die Anbieter nach Wirtschaftlichkeitsverbesserung sowie (politischem) Erfolg des Angebots. Die Rückkehr zu einem anderen Medium wäre in jedem Fall für beide Seiten ein Verlust und die Rückkehr zum Status 
quo. Zweitens wird durch den elektronischen Kanal stärker als bei anderen Kanälen die Art und Weise, in der Dienstleistungen angeboten werden, zum Diskussionsgegenstand. Während klassische Behördendienstleistungen in der Art, wie sie erbracht werden meist nicht mehr infrage gestellt bzw. ertragen werden, gibt es insbesondere bei Web-Dienstleistungen einen hohen Grad an Innovation und Vergleichbarkeit mit einer Vielzahl von anderen Web-Dienstleistungen, die nur ein oder zwei Mausklicks entfernt sind - auch im Bereich E-Government. Drittens ist die Wahrscheinlichkeit groß, dass Drittanbieter auf absehbare Zeit Alternativen für ungenügende E-Government-Services anbieten.

Die Entwicklung von E-Government-Dienstleistungen stand in den ersten Jahren unter dem Primat der angebotsorientierte Strategie. Dadurch konnten relativ schnell eine Vielzahl von Angeboten online gestellt werden, jedoch blieb in vielen Fällen die Ungewissheit im Hinblick auf die Akzeptanz und Zufriedenheit der Nutzer. Da zudem verlässliche Instrumente für eine nachfrageorientierte Strategie bisher kaum entwickelt bzw. erprobt sind, können viele Projekte nicht sicher feststellen, ob bzw. in welchem Maße eine Online-Dienstleistung tatsächlich Bedürfnisse und Interessen der Zielgruppen bedient. Die Risken einer unzureichenden Kundenbindung können für Verwaltungen in ihrer Rolle als Online-Dienstleister auf Dauer aber durchaus bedrohlich werden:

- Take-up und Akzeptanz der Nutzer bleibt hinter den Erwartungen zurück,

- stattdessen weiterhin Nutzung von nicht-internetbasierten Kanälen bzw. von konkurrierenden Angeboten (z. B. auch von privaten Anbietern),

- die Wirtschaftlichkeit (d. h. angestrebtes Kosten-Nutzen-Verhältnis) von E-Government-Projekten ist nicht zufrieden stellend,

- Gefährdung von E-Government-Programmen insgesamt durch nachlassendes Interesse und geringere Förderung.

Befürchtungen und empirische Befunde im Hinblick auf diese Aspekte finden sich national und international, wobei E-Government in Deutschland im internationalen Vergleich ein noch stärkerer Handlungsbedarf bei der Kundenorientierung attestiert wird (vgl. [Acce04], [Zwic04]). Im Folgenden wird auf BundOnline 2005 fokussiert als herausragendes Beispiel einer bundespolitisch gewollten Initiative, die nicht nur eine Reihe von Leistungen des Bundes ins Internet bringen will, sondern sich auf einer übergeordneten Ebene um die Unterstützung des Entwicklungsprozesses von E-Government-Dienstleistungen bemüht.

\subsection{Fallbeispiel: BundOnline 2005}

Mit der Initiative BundOnline hat sich die Bundesregierung verpflichtet, die internetfähigen Dienstleistungen der Bundesverwaltung bis 2005 online bereit zu stellen. Die Online-Bereitstellung der Dienstleistungen des Bundes wird dezentral 
durch die für die jeweiligen Dienstleistungen zuständigen Behörden realisiert. Die Projektgruppe BundOnline unterstützt dabei, indem sie die Gesamtinitiative koordiniert, Synergieeffekte erschließt und so Kosten spart. Zusätzlich fördert sie den Aufbau der erforderlichen Qualifikationen in der Bundesverwaltung z. B. durch die Einrichtung von Kompetenzzentren und Beratung vor Ort. Für die OnlineDienstleistungen werden Softwarelösungen und IT-Dienste als Basiskomponenten zentral bereitgestellt. Perspektivisch sollen sie im Rahmen der übergreifenden Initiative Deutschland-Online auch den Ländern und Kommunen zur Nutzung angeboten werden. Ziel ist es, Mehrfachimplementierungen zu vermeiden. Gleichzeitig wird eine hohe Qualität des angebotenen Dienstleistungsangebots gefördert.

Im Rahmen der E-Government-Initiative BundOnline (vgl. [BMI04]) sollen sechs Basiskomponenten bereitgestellt werden. Diese Basiskomponenten bieten zentral technische Funktionalitäten an, die durch unterschiedliche Dienstleistungen und Behörden genutzt werden können. Sie liefern Technologieplattformen, die - einmal entwickelt - teils identisch oder bedarfsgerecht konfiguriert zur breiten Anwendung in der Bundesverwaltung kommen. Eine wesentliche Eigenschaft der Basiskomponenten ist, dass sie Dienstleistungen unterstützen, selbst aber keine Dienstleistung im Portfolio der Bundesverwaltung darstellen. Vielmehr stellen sie Funktionalitätsblöcke bereit, die Bestandteil sehr vieler Dienstleistungen sind. Dadurch vermindern sie den Realisierungsaufwand und Kosten bei der Umsetzung der BundOnline-Dienstleistungen.

Bei der Umsetzung der Basiskomponenten konnten bereits große Fortschritte erzielt werden. Im Jahr 2003 wurde die Entwicklung der Zahlungsverkehrsplattform abgeschlossen und die erste Version des Government Site Builders (Basiskomponente Content Management System) fertig gestellt und eingesetzt. Für die Basiskomponente Datensicherheit / Virtuelle Poststelle wurde 2004 der Pilotbetrieb aufgenommen. Das Portal www.bund.de und das Formularcenter sind bereits seit der CeBIT 2001 bzw. 2002 online. Mit dem Formular Management System soll künftig auch die medienbruchfreie Verwendung von Online-Formularen ermöglicht werden. Verschiedene Kompetenzzentren, insbesondere die Kompetenzzentren „Datensicherheit“, „Content Management“ und „Zahlungsverkehr“ beraten die Behörden im Vorfeld des Einsatzes von Basiskomponenten und stellen Dokumentationen für die Anwender bereit. Das Kompetenzzentrum „Vorgangsbearbeitung, Prozesse und Organisation" hilft bei der Prozessanalyse und -optimierung sowie bei der Einführung von Vorgangsbearbeitungssystemen.

BundOnline steht für eine Bundesverwaltung, die sich flächendeckend modernisiert, um so den veränderten Erwartungen von Wirtschaft und Gesellschaft gerecht zu werden. Über 100 Bundesbehörden aller Ressorts sind dabei umzuschalten (vom Verwalter zum Dienstleister), so dass Bürgerinnen und Bürger, Wirtschaft, Wissenschaft und auch die Verwaltung selbst entlastet werden, indem sie die Dienstleistungen des Bundes einfacher und schneller in Anspruch nehmen können. 
$\mathrm{Zu}$ Beginn des Projekts lag der Fokus vor allem darauf, Dienstleistungen des Bundes nach vorangegangener Prozessoptimierung in Anlehnung an die Art und Weise, wie sie bis dahin offline angeboten wurden, online zu stellen. In dem Maße, in dem dies gelungen ist, besteht nun die Möglichkeit, ohne großen Zusatzaufwand zu den Online-Angeboten Nutzer-Feedback einzuholen und die Weiterentwicklung verstärkt am Nutzen der „Kunden“ auszurichten. Ziel ist dabei, mit Hilfe der zur Verfügung stehenden Mittel das Optimum an Qualität für die dienstleistenden Verwaltungseinheiten und deren Klienten zu erreichen.

\subsection{Handlungsbedarf}

„Kundenorientierung“ von E-Government-Dienstleistungen als Problem bzw. Zielsetzung umfasst vielfältige Aspekte, entsprechend ist der Handlungsbedarf mehrdimensional. Nicht selten müssen in der betroffenen Behörde Verwaltungsleistungen signifikant umgestaltet werden, um die vollen technischen Möglichkeiten eines Online-Service (bzw. E-Service) auszuschöpfen. Der Entwicklungsprozess bezieht sich also nicht nur auf technische Artefakte, sondern auch auf bereits bestehende Verwaltungsprozesse und die sie bereitstellenden Organisationen:

- organisatorisch / technisch: Wie können Verwaltungen ihre Angebote im Hinblick auf den Kundennutzen verbessern? Wie müssen organisatorische und technische Maßnahmen ineinander greifen?

- projektbezogen / institutionell: Welche Initiativen sind auf Ebene der einzelnen Projekte notwendig? Wie kann bzw. muss institutionell die Basis für eine kundenorientierte Entwicklung nachhaltig unterstützt werden?

- Forschung / Praxis: Wie kann, bei knappen Ressourcen, die Verwaltung ihre eigene Praxis besser in Richtung Kundenorientierung steuern? Welches Wissen ist bei wem notwendig? Was kann die Forschung dabei leisten?

Zwar werden Anliegen und Interessen von E-Government-Kunden mittlerweile stärker untersucht (z. B. [Acce02], [BoFl03], [Gare04]). Jedoch ist eine systematische Vorgehensweise zur kundenorientierten Entwicklung der E-Services bzw. ein entsprechendes Modell zur Projektsteuerung unseres Wissens bisher noch nicht entwickelt und erprobt. Dies wäre jedoch notwendig, um über die Bereitstellung von technischen Basiskomponenten hinaus auch organisatorisch die Entwicklung bzw. Verbesserung von E-Government-Dienstleistungen flächendeckend anleiten zu können. Der vorliegende Beitrag stellt deshalb ein für die Verwaltung geeignetes Modell vor, das beispielsweise im Rahmen der Initiative BundOnline 2005 implementiert werden kann. 


\section{Kundenorientierte Entwicklung von Web- Anwendungen}

Wie bei der anwendungsorientierten Systementwicklung generell, stellt sich auch in den meisten Web-Projekten das Problem, wie die Nutzeranliegen bereits effektiv im Entwicklungsprozess berücksichtigt werden können. Nach Sherrell \& Chen [ShCh01] lassen sich internetbasierte Applikationen in vier Kategorien unterscheiden: (1) Intranets, firmeninterne Netzwerke, (2) Web-Präsenzen oder konventionelle Webauftritte, öffentliche Firmen-Webauftritte als Marketinginstrument, PR und andere Zwecke, (3) Electronic Commerce Systeme, transaktionsorientierte Business-to-Consumer-Webauftritte sowie (4) Extranets, dedizierte Business-to-Business-Netzwerke. Wir konzentrieren uns hier auf die zweite Kategorie, welche die Entwicklung von Web-Portalen einschließt. Diese Systeme bieten eine Sammlung von thematisch zusammengestellten Dienstleistungen. Die meisten EGovernment-Angebote fallen in diese Kategorie, aber auch die mittlerweile nicht mehr überschaubare Zahl von kommerziellen Angeboten, bei denen einzelne Dienstleistungen, wie z. B. Suchmaschinen (Altavista, Google, Yahoo) oder WebMail (Hotmail, Web.de, GMX), zu vollwertigen Portalen aufgebaut wurden.

Im Folgenden leiten wir die kundenorientierte Entwicklung von Web-Anwendungen aus softwaretechnischer Perspektive her und diskutieren deren Herangehensweise im Hinblick auf die Übertragung in die Verwaltung.

\subsection{E-Prototyping}

Im Forschungsgebiet Softwaretechnik steht seit langem die Frage im Mittelpunkt, wie bei anwendungsorientierter Software Benutzer geeignet beteiligt werden können. Evolutionäre und zyklische Entwicklungsmodelle [Flo ${ }^{+} 89$ ], [Somm01], $\left.\left[\mathrm{Abr}^{+} 02\right]\right)$ bieten hierfür wiederkehrende Zeitpunkte an, in denen der aktuelle Entwicklungsstand mit den zukünftigen Anwendern diskutiert wird. Die systematische Einbeziehung von Anwendern wird in diesem Zusammenhang unter dem Stichwort Partizipation diskutiert [ScNa93]. Prototypen haben sich dabei als gutes Hilfsmittel erwiesen, an dem Entwicklungsentscheidungen und deren Auswirkungen diskutiert werden können [Floy84], um so einen Lernprozess auf beiden Seiten zu befördern. Der damit in Zusammenhang stehende Entwicklungsprozess wird als Prototyping bezeichnet.

Bei der Entwicklung von web-basierten Anwendungen stellt sich eine Reihe von Problemen anders bzw. neu, und Annahmen aus dem „traditionellen“ Prototyping sind nicht zwingend richtig für die $\mathrm{e}^{*}$-Welt. Der weiterentwicklete Ansatz des EPrototyping ([Ble $\left.\left.{ }^{+} 02 \mathrm{a}\right],\left[\mathrm{Ble}^{+} 02 \mathrm{~b}\right],\left[\mathrm{Jee}^{+} 03\right]\right)$ trägt den Besonderheiten der Entwicklung von Web-Anwendungen Rechnung. Dabei stützen wir uns auf einen zyklischen Entwicklungsprozess nach Floyd, der aus den Schritten „Projekt/ Versionsetablierung“, „Konstruktion“, „Versionsfreigabe“ und „Nutzung“ besteht. 
Diese haben wir integriert mit den Prototyping-Schritten „Auswahl des Funktionsumfangs“, „Konstruktion“, „Evaluation“ und „Entscheidung über weitere Verwendung" (Abb. 1).

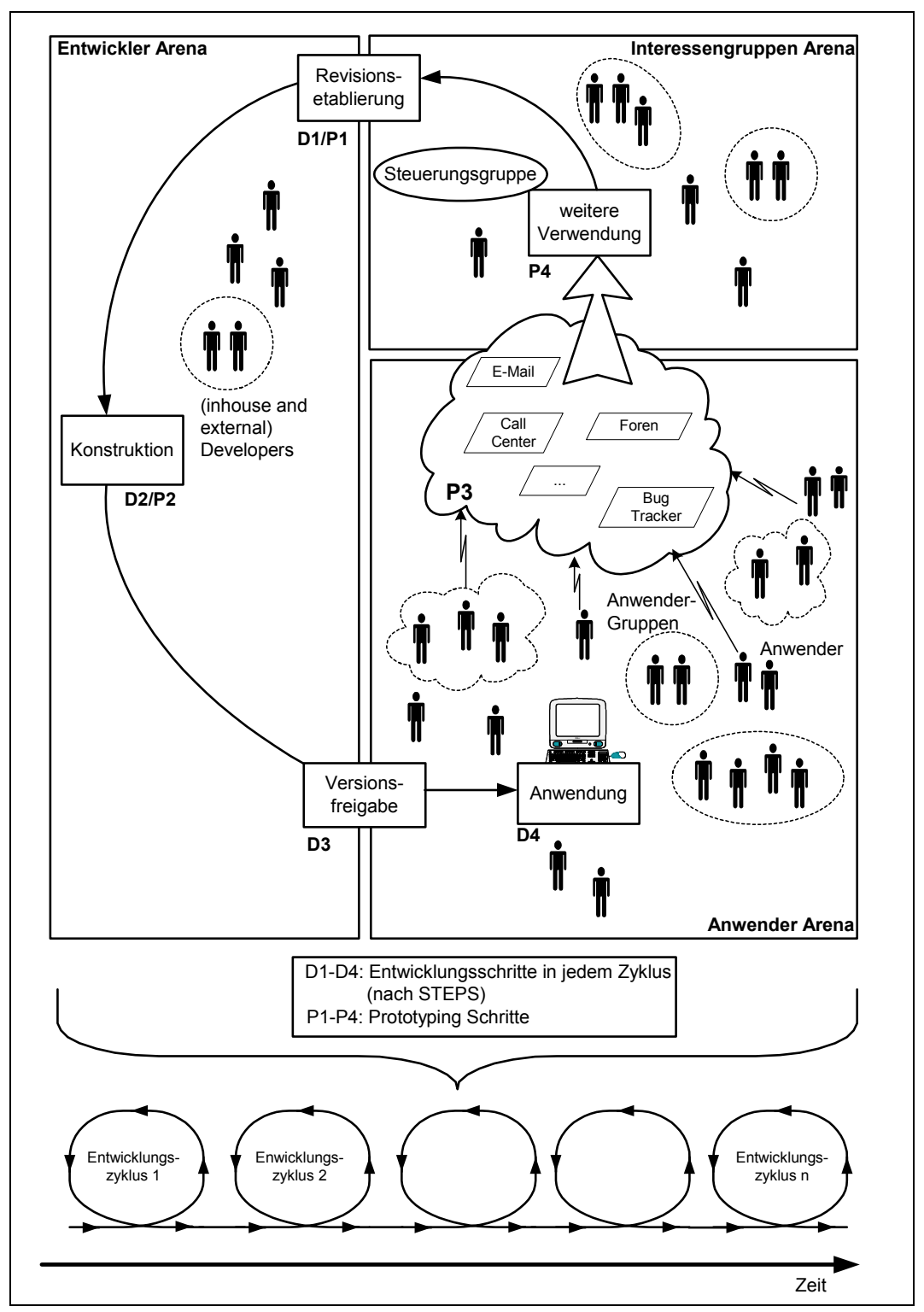

Abbildung 1: Der E-Prototyping-Prozess

Die teilnehmenden Organisationen und Personen betrachten wir mit Hilfe eines Akteursmodell, in dem wir Rollen genauer herausstellen, die im Setting einer 
Web-Anwendung auftreten. Das Feld, in dem die Akteure agieren, unterteilen wir in die folgenden Arenen:

- In der Entwickler-Arena sind die Akteure zusammengefasst, die mit der Entwicklung der späteren Web-Anwendung beschäftigt sind. Hierzu zählen neben klassischen Software-Entwicklern nun zusätzlich z. B. Screen-Designer, die grafische Gestaltung liefern, und Redakteure, die dem Medium angemessene Texte produzieren. Andere Mitglieder dieser Arena sind Vertreter der Organisationen, deren Produkte eingesetzt werden, wie z. B. Datenbanken, Applikations- und Webserver. Die Aufgabe der Akteure in der Entwickler-Arena ist es, eine geeignete Version für die spätere Anwendung zu konstruieren. Im Entwicklungsprozess für Web-Anwendungen treten neue Rollen in Erscheinung, die bei klassischen Software-Projekten bisher nicht im Vordergrund standen. Dazu zählen z. B. Designer und Lieferanten von Inhalten (Content), aber auch freiwillige Nutzer. Eine wichtige neue Rolle nimmt der Bereitsteller (Provider) von Hardware, Standard-Software und Internet-Anbindung ein, der bei WebAnwendungen im Allgemeinen unumgänglich ist.

- In der Anwender-Arena sind die verschiedenen Gruppen von Personen zusammengefasst, die die bereitgestellte Applikation anwenden. Die von außen an die Organisation herantretenden Anwender von Web-Dienstleistungen lassen sich als eine sehr heterogene Gruppe charakterisieren, d. h. in vielen Anwendungskontexten müssen Personen bedient werden, die sowohl sehr wenig Fachwissen über die Anwendung haben als auch solche, die sich besser als professionelle Anwender charakterisieren lassen. Im Allgemeinen sind diese Anwender eine Gruppe von unbekannten Personen, die trotz ihrer Nutzung nur teilweise bekannt werden. Die Anwender haben die Eigenschaft von Kunden, d. h. sie nutzen eine Dienstleistung nur so lange, wie sie ihren Ansprüchen gerecht wird. Gibt es einen anderen Anbieter, der eine ähnliche Anwendung zur Verfügung stellt, wird verglichen, gefordert und ggf. gewechselt. Darüber hinaus gibt es die Anwender in der bereitstellenden Organisation. Diese sind alle bekannt und erreichbar. Aber auch hier liegen unterschiedliche Profile in der Nutzung zugrunde. In der Anwender-Arena finden wir deshalb typischerweise eine sehr heterogene Personengruppe vor. „Stille“ Anwender, institutionelle Anwender, Anwender unterschiedlicher Aus- und Vorbildung, sowie aktive Anwender sind Beispiele für die Verschiedenartigkeit dieser Gruppe. Im Zentrum dieser Arena steht die Nutzung der Web-Anwendung. Je nach Charakter der konkreten Anwender kann Feedback über unterschiedliche Kanäle gesammelt werden. Bewährt haben sich insbesondere: Help-Desk, E-Mail, Diskussionsforen, Call-Center sowie die statistische Auswertung des IT-Betriebs (Seitenabrufe etc.). Für das E-Prototyping entscheidend ist, die Möglichkeiten zur Rückmeldung vielfältig, einfach und möglichst unmittelbar an der Nutzungssituation bereitzustellen.

- In der Interessengruppen-Arena finden sich Vertreter aus den verschiedenen beteiligten Organisationen wieder, die für die Entwicklung einer Web- 
Anwendung relevant sind. Hierzu zählt das Management der initiierenden Organisation, Vertreter der Vertragspartner (z. B. Inhaltslieferanten), Vertreter der zukünftigen Nutzergruppen (z. B. Betriebsrat). Ihre Aufgabe ist es, über den Umfang einer ersten bzw. folgenden Version zu entscheiden. Im Folgenden fließen dann Erfahrungen aus der Nutzung in die Bewertung der aktuellen Version ein und werden als Grundlage für die Entscheidung in der Interessengruppen-Arena weiter verwendet, wenn es um eine Folge-Version geht.

Die Mitglieder der drei Arenen nehmen eine jeweils eigene Sicht auf die Software ein. So sind z. B. die Software-Entwickler häufig daran interessiert, ihre bevorzugte Technologie einzusetzen oder die Screen-Designer legen Wert auf ein ausgefallenes Design. Aus der Gruppe der Anwender wird vielfach die Erwartung geäußert, dass neue Versionen und speziell Fehlerbehebungen zügig verfügbar gemacht werden. Ganz anders sind die Zielsetzungen von Vertretern der Interessengruppen-Arena. Dem Auftraggeber und dem Management steht ein Return-onInvestment im Vordergrund, dem Benutzervertreter womöglich die Benutzbarkeit der Software und die damit verbundenen Freiheitsgrade.

Das Vorgehensmodell des E-Prototypings sieht folgenden Prozess vor: In der Steuerungsgruppe wird über den Funktionsumfang der Software entschieden. Für eine erste Version wird eine Teilmenge der Funktionen als „Kernsystem“ [ $\mathrm{Kra}^{+} 97$ ] bestimmt. Der Auftrag an die Entwickler wird erteilt, dieses Kernsystem mit den angemessenen Technologien zu konstruieren. Innerhalb eines kurzen Zeitraums (ca. 3-6 Monate) wird eine Version erstellt und für die Anwendung freigegeben. In der Anwender-Arena findet die Nutzung der Software-Version statt und Feedback über die Nutzung wird über verschiedene Kanäle gesammelt. Die Auswertung des Feedbacks wird in einem nächsten Schritt der Steuerungsgruppe zugeführt. Diese befindet bei der nächsten Version darüber, welche Teilvorhaben in der nächsten Version zusammengefasst werden.

Die Vorteile des E-Prototyping-Prozesses für die Entwicklung von Web-Anwendungen sind vor allem folgende:

- Systematisch gesammeltes Feedback ermöglicht, aktuelle und stimmige Anforderungen zu ermitteln und an die Gestalter und Konstrukteure zu übermitteln.

- Kurze Entwicklungszyklen unterstützen die Berücksichtigung der gerade bei Web-Projekten häufigen Änderungen der Anforderungen und ihre Umsetzung in regelmäßige Releases.

- Wissenslücken aufzeigt und Know-how unter den Akteuren verbreiten.

Mit Hilfe dieses Vorgehensmodells kann ein besonderes Problem, nämlich dass Anforderungen der Web-Anwender erst im Verlauf deutlich werden, frühzeitig und kontinuierlich adressiert werden. Von einem Modell, das bereits in der Praxis genutzt wurde, um insbesondere die Effektivität der Softwareentwicklung zu verbessern (vgl. [Pap $\left.\left.{ }^{+} 01\right],\left[\mathrm{Flo}^{+} 04\right]\right)$, wird im Folgenden ausgegangen, damit ein 
angemessener Prozess für die kundenorientierte Entwicklung von E-GovernmentDienstleistungen konzipiert werden kann.

\section{2 Übertragung auf Verwaltungsdienstleistungen}

Beim Perspektivenwechsel von der Softwaretechnik zur Entwicklung von EGovernment-Angeboten ist vor allem zu beachten, dass die Verwaltungsakteure als Adressaten der Vorgehensweise sich nicht primär als Entwickler verstehen, sondern vielmehr von der Perspektive von Projektmanagement und Interessengruppen aus Anstöße zur Verbesserung liefern wollen. Aus diesem Grund ist der oben eingeführte Begriff E-Prototyping (der bei IT-Experten unmittelbar eine Reihe von Assoziationen weckt) nicht weiter hilfreich, und im nachfolgenden Abschnitt wird diesem Perspektivenwechsel entsprechend Rechnung getragen. Bei der Entwicklung einer E-Government-spezifischen Vorgehensweise sind im Vergleich zum Web-Engineering darüber hinaus weitere Besonderheiten zu berücksichtigen (zu den Besonderheiten der Verwaltung siehe z. B. [Lenk99], [Len $\left.{ }^{+} 02\right]$ ):

- Der Fokus liegt nicht auf der Entwicklung von funktionsorientierten Softwarekomponenten, sondern von internetbasierten Softwareanwendungen, die einen aus der sozialen Welt begründbaren Nutzen für Anwender darstellen.

- E-Government-Services müssen (jeweils abhängig von Gegenstand und Art der Leistung) das gesamte Spektrum zwischen kleineren Zielgruppen mit bekannten Nutzern und nicht näher eingrenzbaren Nutzergruppen (ggf. Gesamtbevölkerung) abdecken.

- Der Inhalt von E-Government-Leistungen ist nur in den wenigsten Fällen einfach standardisierbar: sachgerechte Information und Bearbeitung (Transaktion) erfordert häufig individuelle Fallunterscheidungen bzw. Einzelfallentscheidungen, weswegen sich mehr oder weniger große Prozessanteile bei der Bearbeitung von Bürgeranliegen nicht automatisieren lassen.

- Im Vergleich zu E-Services im kommerziellen Bereich unterliegen Dienstleistungen im E-Government höheren Anforderungen hinsichtlich Sicherheit, Datenschutz und Gesetzeskonformität.

- Die Anwendungswelt von E-Government-Services ist durch eine vergleichsweise hohe Regelungsdichte und eine große Zahl organisierter Akteure (Verbände) gekennzeichnet.

- Die vielfältigen Nutzendimensionen des Verwaltungshandelns bei gleichzeitig fehlender Tradition der wirtschaftlichen Bewertung erschweren die Ausrichtung der Dienstleistungsentwicklung an Wirtschaftlichkeitsüberlegungen.

- Die technischen Grundlagen für E-Services werden in hohem Maß durch Outsourcing von IT-Dienstleistungen entwickelt und bereitgestellt, wodurch die Steuerungsfähigkeit der Verwaltung zusätzlich erschwert ist. 
Vor diesem Hintergrund skizzieren wir im Folgenden eine Vorgehensweise, die die Steuerungsfähigkeit von E-Government-Projekten verbessert und dabei eine systematische Ausrichtung auf die Nutzer der Online-Angebote ermöglicht.

\section{Neues Steuerungsmodell für E-Government- Projekte}

Der Begriff „neues Steuerungsmodell“ verweist sowohl auf die Ebene der Steuerung von Einzelprojekten als auch auf die Ebene der institutionellen Verankerung von E-Government-Projektsteuerung. Basierend auf den Erkenntnissen und Erfahrungen der Softwaretechnik sowie unter Berücksichtung der Besonderheiten der Verwaltung skizzieren wir eine Vorgehensweise für die Entwicklung von EGovernment-Dienstleistungen, die neben den bisher Beteiligten darüber hinaus den Dienstleistungsnehmern eine aktive Rolle einräumt. Mit dem bewussten Rekurs auf die Konzepte des New Public Management soll betont werden, dass eine Orientierung am Neuen Steuerungsmodell notwendig ist, um die Professionalisierung der Entwicklung von E-Government-Dienstleistungen sowohl auf der Projektebene als auch auf der Ebene der Institutionen zu befördern.

\subsection{Vom New Public Management zur Kundenorientierung}

Die Prinzipien des Neuen Steuerungsmodells (NSM) sind im Wesentlichen (vgl. [KGSt93], [KGSt94], zitiert nach www.olev.de/n/nsm.htm):

- Steuern statt Rudern: Leistungen sind zu gewährleisten und zu kontrollieren, nicht alles ist selber zu machen.

- Resultate statt Regeln: Orientierung an Ergebnissen und Kosten statt Fixierung auf Verfahren und Regeln.

- Eigenverantwortlichkeit statt Hierarchie: klare Zuweisung und ungeteilte Verantwortung für die Ressourcen.

- Wettbewerb statt Monopol: Ermittlung von Kosten und Qualitäten öffentlicher Leistungen im Vergleich zu anderen Anbietern.

- Motivation statt Alimentation: Neubestimmung der „Ressource Personal“, die weit über eine Änderung des Dienstrechts hinausgeht.

In diesen Prinzipien ist von Kundenorientierung noch keine Rede, was auf die primäre Motivation des NSM zur Verbesserung der Leistungsfähigkeit der Verwaltung bei gleichzeitiger Kostenreduktion zurückzuführen ist. In der Verwaltungsliteratur wird jedoch übereinstimmend die „Einbeziehung der Bürger in den Modernisierungsprozess“ als wesentliches Element dargestellt, dessen Umsetzung 
in die Praxis aber (zunächst) stark vernachlässigt wurde [BoKi97a]. Die Charakterisierung der Verwaltung als Dienstleister greift zu kurz, verstünde man darunter nur einen Akteur, der seine Leistung am Bedürfnis des Kunden ausrichtet schließlich tritt die Verwaltung dem Bürger z. B. auch als Ordnungsmacht gegenüber und verfolgt zudem weitere Ziele, die sich am Gemeinwohl orientieren (das dem Anliegen des Einzelnen ggf. widersprechen kann).

Dennoch ist der Zusammenhang von (Dienst-)Leistung, Kundenorientierung und NSM essentiell: die Orientierung an Ergebnissen und Kosten (statt Fixierung auf Verfahren und Regeln) sowie die Ermittlung von Kosten und Qualitäten öffentlicher Leistungen (gerade auch im Vergleich zu anderen Anbietern) sind nur sinnvoll und möglich, wenn die Verwaltung den Bürgern, Unternehmen und anderen Interaktionspartnern auf „Augenhöhe“ [BoKi97b] begegnet - ein Perspektivenwechsel, der nicht nur bei Verwaltungsmitarbeitern, sondern auch bei den Adressaten ihrer Leistungen ein Lernprozess erfordert (vgl. ebd.).

Ziel der hier skizzierten Vorgehensweise ist deshalb, alle Beteiligten bei der Entwicklung und Nutzung von E-Government-Dienstleistungen als Partner in einen systematischen Zusammenhang zu bringen, der den technischen Entwicklern und Prozessgestaltern aktuelle nachfrageorientierte Vorgaben liefert, den Nutzern umfangreiche Möglichkeiten zur Artikulation ihrer Nutzungsanliegen bietet und der Verwaltung eine strategische und gleichzeitig kleinschrittige Steuerung der internet-basierten Dienstleister-Kunden-Beziehung sowie der entsprechenden Allokation von Ressourcen ermöglicht.

\subsection{Vorgehen bei der Entwicklung von web-basierten E-Government-Dienstleistungen}

Im Mittelpunkt des neuen Steuerungsmodells für die Entwicklung von E-Government-Dienstleistungen steht die Verwaltungsleistung, die bereits in vielen Fällen ohne technische Unterstützung existiert und im Rahmen des technischen Fortschritts und unter Ausnutzung von Synergieeffekten über einen zusätzlichen Kanal - in vielen Fällen das Web - angeboten werden soll. Dabei besteht der E-Service aus einer Reihe verschiedenartiger Bestandteile. Neben den Software-Komponenten und einer für den Betrieb erforderlichen IT-Infrastruktur gehören dazu die Inhalte, die verfügbar gemacht werden sollen, sowie die notwendigen Einrichtungen für Service rund um den neuen Kanal (Help-Desk, Support etc.).

Das hier vorgestellte Modell (siehe Abb. 2) besteht aus einem mehrfach zu durchlaufenden Zyklus mit drei Abschnitten: Planung, Konstruktion und Anwendung. Im Zentrum dieser Abschnitte steht die Verwaltungsleistung, an der sich die EService-Implementation ausrichtet. Der Übergang zwischen den Abschnitten wird über Artefakte koordiniert, die jeweils die Grundlage für den folgenden Abschnitt sind. Die Entwicklung betrifft dabei sowohl die Verwaltungsleistung als auch die aufzubauenden bzw. weiter zu entwickelnden IT-Leistungen, da im Entwicklungs- 
prozess Wechselwirkungen unausweichlich sind. Im Folgenden werden die Abschnitte erläutert sowie der essentielle Feedback-Prozess detailliert beleuchtet:

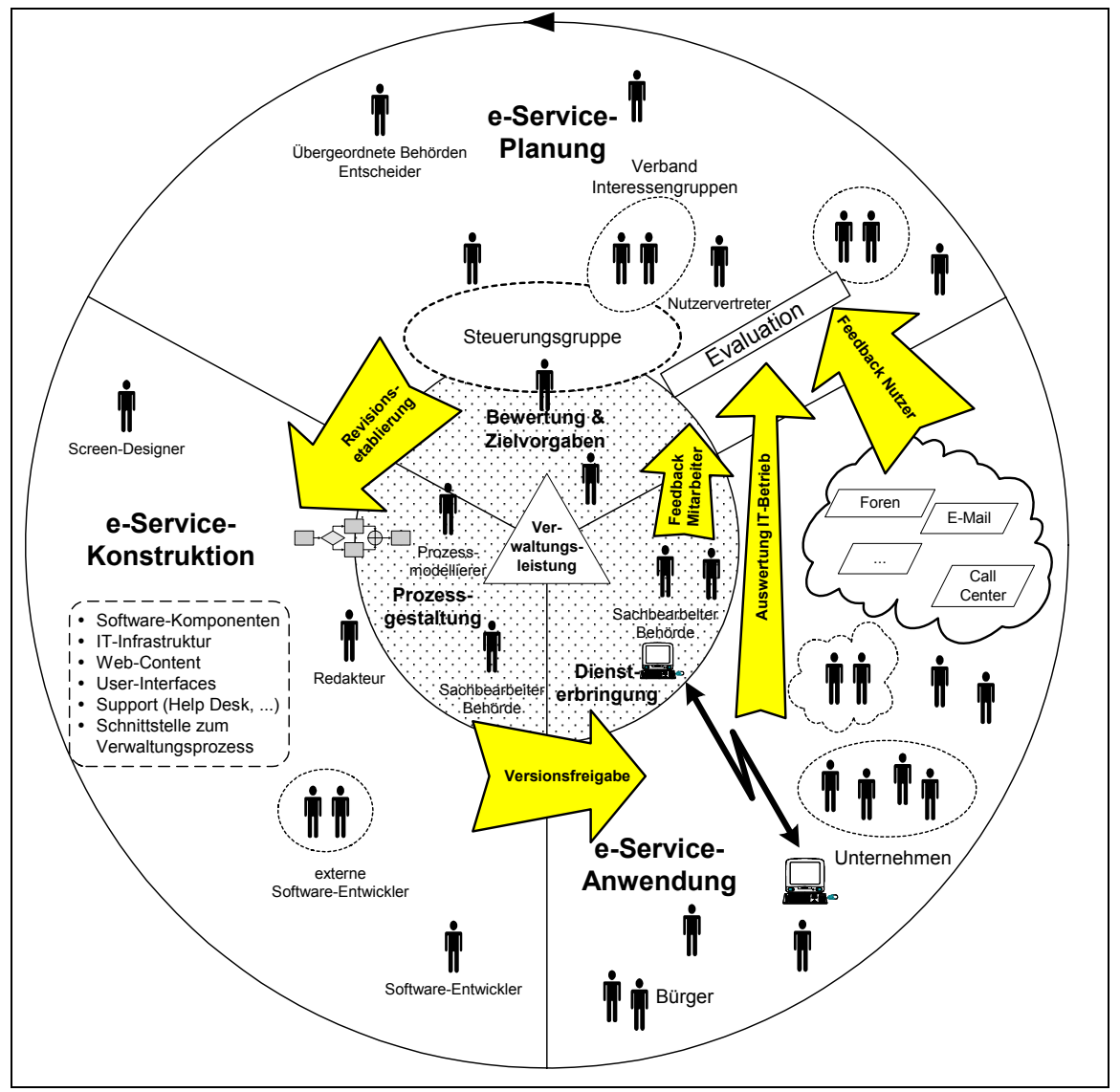

Abbildung 2: Steuerungsmodell für die kundenorientierte Entwicklung von E-GovernmentDienstleistungen (innerer Kreis: Entwicklungszyklus der Verwaltungsleistung)

\section{E-Service-Planung}

Die Initiierung des kundenorientierten Entwicklungsprozesses beginnt im Abschnitt der E-Service-Planung. Auf der Grundlage von Zielvorgaben und einer ersten Bewertung wird von der Steuerungsgruppe eine Projektetablierung durchgeführt. Bereits bekannte relevante Personenkreise können in die Steuerungsgruppe einbezogen werden, um den Umfang einer ersten Systemversion zu bestimmen und eine Planungsgrundlage für den nächsten Abschnitt zu erstellen. Zu den Personen gehören Vertreter von Entscheidungsträgern aus übergeordneten Behörden und von Verbänden und Interessengruppen genauso wie Vertreter aus 
bereits im Vorfeld bekannten Nutzergruppen. Zielvorgaben aus der Politik und Verwaltung werden von der Arbeit der Steuerungsgruppe berücksichtigt.

Tritt der Entwicklungsprozess wiederholt in die E-Service-Planung ein, dienen die zusätzlich gewonnenen Informationen über die Nutzung (s. u.) als Grundlage für weitere Entscheidungen. Diese Erfahrungen können auch dazu führen, dass neue Mitglieder in die Steuerungsgruppe aufgenommen werden (bisher unbeachtete Nutzergruppen oder Interessenvertreter) oder bisherige ausscheiden. Ergebnis ist jeweils eine Revisionsetablierung (Etablierung einer Folgeversion), die in einer Systemspezifikation festgehalten wird und auf dem existierenden System aufsetzt.

\section{E-Service-Konstruktion}

Das durch die E-Service-Planung mithilfe der Steuerungsgruppe etablierte Projekt (bzw. die etablierte Revision) geht in die E-Service-Konstruktion und wird in $\mathrm{Zu}-$ sammenarbeit mit Behördenvertretern, die Fachwissen über den relevanten Prozess einbringen können, umgesetzt. Notwendiges Fachwissen auf den vielfältigen technischen Gebieten (Datenintegration, Sicherungsmechanismen, Verteilung, usw.) wird in der Konstruktion durch Spezialisten, z. B. Software-Entwickler, eingebracht. Der Besonderheit von E-Government-Projekten, dass Softwareentwickler hier zumeist externe Mitarbeiter sind, trägt der Prozess dadurch Rechnung, dass er ausreichend kleinschrittig vorgeht und die notwendigen Kommunikationsund Koordinationsmittel einbezieht. Eine dem Medium gerechte Umsetzung stellen Vertreter aus den entsprechenden Professionen sicher (z. B. Screen-Designer und Redakteur). Im Abschnitt der Konstruktion findet ein dem Entwicklungsgegenstand angemessener softwaretechnischer Entwicklungsprozess statt. Empfehlenswert sind z. B. agile Methoden, die sich besonders gut für kurze Zyklen eignen. Erreicht der E-Service den in der Etablierung festgelegten Umfang, der im ersten Durchlauf dem Kernsystem (s. o.) entspricht, wird die Version freigegeben und steht somit den Bürgern bzw. anderen Zielgruppen zur Nutzung bereit.

Die Einführung von Informationstechnik kann Rückwirkungen auf den existierenden Verwaltungsprozess haben. In diesem Sinne umfasst die E-Service-Konstruktion ebenfalls die (Weiter-)Entwicklung des Verwaltungsprozesses und die damit verbundene Anpassung in den ausführenden Institutionen. Die Einsatzvorbereitung muss deshalb nicht nur die Erreichbarkeit und Nutzbarkeit des neuen Kanals bzw. der neuen Dienstleistung für Endanwender ermöglichen (dies ist speziell für web-basierte Anwendungen relativ leicht umsetzbar), sonder muss auch der Anbindung an die entsprechenden Sachbearbeiter in der Behörde gerecht werden.

\section{E-Service-Anwendung}

Personen aus der adressierten Nutzergruppe können von nun an den E-Service anwenden und so ihr Kundenverhältnis zum Dienstbetrieb über einen neuen Kanal ausüben. Auf Seiten der Behörde ist der zusätzliche Kanal bzw. die neue Dienstleistung in den Arbeitsablauf integriert worden. Im Rahmen der E-Service- 
Nutzung fällt vielfältiges Feedback auf beiden Seiten der Nutzung an. Anwender können dafür die bereitgestellten und (prominent beworbenen) Feedback-Medien (z. B. Diskussionsforen) verwenden. Darüber hinaus ist es sinnvoll, die Nutzung des E-Service im Rahmen der durch Datenschutzbestimmungen gegebenen Möglichkeiten technisch auszuwerten. Hier können wertvolle Informationen über Abbrüche, Fehlersituationen und Nutzungsfrequenz gewonnen werden. Auf der Seite des Dienstanbieters (der Behörde) können ebenfalls Rückmeldungen über die Nutzungserfahrungen bei den betroffenen Behördenmitarbeitern gesammelt werden. Feedback aus beiden Gruppen und die Auswertung des IT-Betriebs zusammen fließen als verdichtete Informationen in eine Evaluation ein, die dem nächsten E-Service-Planungs-Abschnitt als Grundlage dient. Die Rohdaten werden zu einem kontinuierlich erstellten Evaluationsdokument mit quantitativen und qualitativen Information verarbeitet, welches der Steuerungsgruppe eine Grundlage für die Entscheidungen zur Weiterentwicklung liefert.

Im Rahmen des Einsatzes des E-Services schärft sich das Verständnis über die Nutzergruppe derart, dass über Quantität und Ausprägung der Nutzer sicherere Aussagen gemacht werden können. Der Aufgabe der Feedback-Auswertung kommt deshalb eine besondere Rolle zu. Die sorgfältige Beschäftigung mit den Nutzern sowohl auf der Seite der Bürger als auch auf der Seite der Behördenmitarbeiter ermöglicht es, ein ausdifferenziertes Bild vom Anwenderkreis zu erarbeiten, welches die Entscheidungen über Weiterentwicklungen erleichtern.

\section{Feedback-Auswertung als Schlüsseltätigkeit}

Das Einholen, Beantworten und Bewerten von Feedback über die E-GovernmentDienstleistung wird zu einer Schlüsseltätigkeit im Entwicklungsprozess. Dabei ist es notwendig, die verschiedenen Kanäle passend der vermuteten Nutzergruppe angemessen auszuwählen. Es bieten sich spezielle E-Mail-Adressen, E-MailListen, (web-basierte) Diskussionsforen, Community Systeme usw. an. Dabei ist zu beachten, dass alle auf Seiten der Bürger angebotenen Kanäle nicht nur betrieben werden, sondern auch regelmäßige Reaktion zeigen müssen. Dies kann z. B. durch Online-Redakteure geschehen, die sich aktiv um Antworten auf gestellte Fragen kümmern und wiederkehrende Fragen und Antworten sammeln und separat zur Verfügung stellen. Die Qualität der Rückmeldung von Seiten der Anwender wird maßgeblich dadurch bestimmt, in welcher Qualität die Antworten im Feedback-Medium sind. Es ist zu beachten, dass die Kommunikation der Verwaltung mit dem Bürger besonderen rechtlichen Rahmenbedingungen unterliegt und dass besonderes Augenmerk auf den datenschutzrechtlich korrekten Umgang mit dem Nutzerfeedback gelegt werden muss.

Unabhängig von den angebotenen Kanälen sollte die Behandlung der Rückmeldungen gleichartig vorgenommen werden, d. h. Eingänge über unterschiedliche Medien sollten mit derselben Sorgfalt bearbeitet werden. Dies umfasst insbesondere die Beantwortung bzw. Bereitstellung von häufig gestellten Fragen (FAQ) 
zusammen mit Antworten. Hier bietet sich eine Vorgangsverfolgung (Stichwort Help-Desk mit sog. „Trouble-Ticket") an.

Bei der gesamten Organisation des Feedbacks muss im Auge behalten werden, dass E-Government-Dienstleistungen vielfach an zwei Enden durch Nutzergruppen bedient werden. Viele Verfahren erfordern beispielsweise, dass nach der Einsendung durch den Antragsteller Sachbearbeiter in der Behörde fallspezifische Entscheidungen treffen. Die Verwaltungsmitarbeiter dürfen bei der E-ServiceEntwicklung deshalb nicht vergessen werden. Sie sind Nutzer einer Software und bedürfen derselben Betreuung.

Indirektes Feedback kann insbesondere bei Web-Anwendungen zusätzlich durch Auswertung der technischen Nutzung gesammelt werden. Log-Dateien bieten Auskunft über die Häufigkeit der Anwendung, Navigationspfade und Abbrüche. Diese Form der Auswertung sollte bereits während der Konstruktion berücksichtigt werden, um entsprechende technische Vorkehrungen zu treffen (z. B. SessionVerwaltung, eigene (anonyme) Nutzungszähler, interne Anwendungen für die Auswertung). Bei allen Auswertungen sind die geltenden Datenschutzbestimmungen zu berücksichtigen und ggf. streng auszulegen. Eine transparente Dokumentation der Auswertung gegenüber den Anwendern hilft Vertrauen aufzubauen.

Neben der inhaltlichen Auswertung des gesammelten Feedbacks sind äußere Eigenschaften von Interesse, um die Feedback-Kanäle der Zielgruppe angemessen zu gestalten. Die Bewertung von Feedback sollte deshalb zusätzlich auf den Ebenen Kanal, Frequenz und Thema erfolgen. Welche Kanäle werden akzeptiert bzw. vorwiegend gewählt? Wie häufig werden dieselben Fragen gestellt? Welche Inhalte stehen im Mittelpunkt der Anfragen bzw. Rückmeldungen? usw. Diese Fragen helfen, die Gesamtheit der gesammelten Nutzungsdaten für Entscheidungen im Bereich der Steuerungsgruppe vorzubereiten.

Die Kundenorientierung (vgl. 3.1) dieses Entwicklungs-Prozesses macht sich an mehreren Stellen fest. Zum einen bietet das Vorgehen die Möglichkeit, mit Hilfe von kurzen Zyklen geeignete zügige Versionsfortschritte umzusetzen. Nutzer bekommen in regelmäßigen Abständen neue Versionen zur Verfügung gestellt, die kundenorientierte Änderungen reflektieren. Zum anderen ergibt sich über die Auswertung der Nutzung und die Kommunikation mit den Nutzern maßgeblicher Einfluss auf die Gestaltung der nächsten Version. Beteiligung der verschiedenen Nutzer- bzw. Interessengruppen in der Steuerungsgruppe ermöglicht diesen direkten Einfluss auf Umfang und Zeitrahmen weiterer Versionen. Eine Version umfasst dabei sowohl die spezifische Software für die Verwaltungsdienstleistung als auch die Verwaltungsleistung selbst, die in diesem Entwicklungsprozess ggf. Änderungen widerfährt. Auslöser für Änderungen auf verschiedenen Seiten ist die im Prozess angelegte Kommunikation, die zu einem allseitigen Lernprozess führt. 


\subsection{Anwendung des Steuerungsmodells}

Das hier skizzierte neue Steuerungsmodell zur kundenorientierten Entwicklung von E-Government-Dienstleistungen adressiert die oben aufgeworfenen handlungsleitenden Fragen (Abschnitt 2.2):

- Den als Dienstleister agierenden Verwaltungen wird eine Herangehensweise angeboten, wie sie ihre Angebote im Hinblick auf den Kundennutzen in einem zyklischen Prozess nachhaltig verbessern können. Insbesondere für die Konstruktion und Anwendung von E-Service wird aufgezeigt, wie organisatorische und technische Maßnahmen jeweils ineinander greifen müssen und wann welcher Steuerungsbedarf besteht.

- Das Steuerungsmodell kann jederzeit im Rahmen einzelner Projekte angewandt werden. Für größere und verteilte Verwaltungen mit einer Vielzahl von E-Government-Projekten bietet es zudem einen übergreifenden Bezugsrahmen, um auch institutionell die Basis für eine kundenorientierte Entwicklung von EGovernment-Dienstleistungen nachhaltig zu unterstützen: durch geeignete zusätzliche Infrastrukturkomponenten (u. a. Feedback-Kanäle, Kommunikationsplattformen, Evaluationsinstrumente), Auswertung und Vermittlung von BestPractices, Verwaltungsrichtlinien, Trainings von Verwaltungsmitarbeitern und Beratern, entsprechende Vertragsgestaltung mit IT-Zulieferern etc.

- Die Entwicklung von E-Government-Dienstleistungen ist gekennzeichnet durch die Beteiligung vieler Akteure sowie durch knappe Ressourcen und vergleichsweise geringe technische Kompetenz auf Seiten der Verwaltungen. Das hier skizzierte Steuerungsmodell trägt diesem Rechnung durch einen leichtgewichtigen Entwicklungsprozess (d. h. kurze Zyklen, überschaubare Arbeitspakete, relativ geringe Investitionen), der die für die Dienstleistung verantwortliche(n) Verwaltung(en) ins Zentrum stellt, um von da aus die eigene Praxis besser in Richtung Kundenorientierung zu steuern. Das Modell, das auf jahrelange Forschung und Praxis in der Softwaretechnik zurückgeht, macht deutlich, welches Wissen (über Dienstleistungsprozess, Kundenanliegen, Technikunterstützung) für die Steuerungsfähigkeit insgesamt und bei den einzelnen Akteuren in den verschiedenen Schritten notwendig ist. Über den initialen Transfer und die domänenspezifische Weiterentwicklung dieses oder anderer Vorgehensweisen hinaus ist die E-Government-Forschung nun selbst gefordert, die praktische Relevanz solcher Ansätze im Hinblick auf „Kundenorientierung“ (hier: Verwaltungen) empirisch zu überprüfen, kritisch zu reflektieren und durch praxisorientierte Vorschläge weiter zu unterstützen.

Die Elemente des vorgestellten neuen Steuerungsmodells für einen E-Government Entwicklungsprozess orientieren sich am etablierten E-Prototyping und den empirischen Erfahrungen, die u. a. im Projekt hamburg.de gesammelt wurden. Basierend auf den Erfahrungen in Einzelprojekten greift der hier vorgestellte Prozess die wesentliche Rolle des Feedbacks auf und adressiert mit den bereits erprobten 
Elementen das besondere Anwendungsfeld der Verwaltungsleistungen. Die Prinzipien des NSM (vgl. 4.1) sind dabei auch handlungsleitend für das Steuerungsmodell zur kundenorientierten Entwicklung von E-Government-Dienstleistungen:

- Steuern statt Rudern: Aus Sicht der Verwaltung als E-Government-Anbieter ist entscheidend die Steuerung und Supervision des gesamten Entwicklungszyklus (Abb. 2) - die einzelnen Aktivitäten darin können bzw. müssen von externen Partnern übernommen werden.

- Resultate statt Regeln: Die weitere Planung sollte sich stets flexibel an Ergebnissen und Kosten der einzelnen Entwicklungsabschnitte orientieren.

- Eigenverantwortlichkeit statt Hierarchie: Das Steuerungsmodell definiert klare Übergänge zwischen den Entwicklungsabschnitten, innerhalb derer die beteilgten Akteure eigenverantwortlich agieren sollten. Die Gesamtsteuerung erfolgt durch periodische Evaluation und Zielvorgaben, nicht durch einzelne Direktiven im Projektalltag.

- Wettbewerb statt Monopol: Die Orientierung am „Online-Kunden“, d. h. am Bürger oder Unternehmen als Web-Anwender, koppelt Erkenntnisse über den relativen Nutzen (im Vergleich zu anderen Angeboten oder Kanälen) in den Entwicklungsprozess zurück. Auch innerhalb der Verwaltung sollte im Rahmen des Steuerungsmodells ein Wettbewerb um „Best Practice“ stattfinden.

- Motivation statt Alimentation: Eine positive und konstruktive Grundhaltung der Beteiligten (sowohl hinsichtlich neuer Technologien als auch der Dienstleistungsbeziehung zwischen öffentlicher Verwaltung und ihren Partnern) kann nicht immer vorausgesetzt werden. Das vorgestellte Steuerungsmodell begünstigt diese Grundhaltung und kann so zu einer systematischen Entfaltung der professionell vorhandenen Potenziale beitragen.

Anwendungsfeld für das oben skizzierte Steuerungsmodell kann jede Organisation sein, die den Entwicklungsprozess von E-Government-Dienstleistungen systematisch und nachhaltig am Nutzen ihrer „Kunden“ orientieren will (wie z. B. die Initiative BundOnline). In jedem Fall wären zunächst Pilotvorhaben vorzusehen (einschließlich deren Auswertung und Aufbereitung als Best-Practice-Beispiele) für ausgewählte Dienstleistungen und auch für die Weiterentwicklung und Präsentation des Dienstleistungsportfolios insgesamt. Positive Effekte sind sowohl für die betreffenden Dienstleistungen zu erwarten als auch auf der Ebene des Organisationslernens, d. h. die Beförderung einer Organisationskultur, die „Kundenorientierung“ bei der Durchführung von E-Government in der Praxis selbstverständlich berücksichtigt.

Zusammenfassend lässt sich feststellen, dass die Kundenorientierung im Rahmen von E-Government-Projekten und -Initiativen zwar erheblich an Bedeutung gewonnen hat, dass aber über Umfragen hinaus noch keine Instrumente für die Praxis vorliegen, um die Anliegen der E-Government-Nutzer im Rahmen der Entwicklung von Online-Dienstleistungen effektiv zu berücksichtigen. Mit dem hier 
vorgestellten neuen Steuerungsmodell, das auf den jahrelang gewachsenen Erkenntnissen und Erfahrungen der Softwaretechnik beruht, werden neue Herangehensweisen zur Verbesserung der Kundenorientierung von E-GovernmentDienstleistungen eröffnet bzw. systematisch zusammengeführt. Die Auswertung von Pilotvorhaben muss dabei noch zeigen, worin ggf. Probleme bei der Umsetzung in den einzelnen Projektabschnitten liegen und ob die gewählten Maßnahmen tatsächlich geeignet sind, das Bewusstsein der am Entwicklungsprozess Beteiligten im Hinblick auf Kundenorientierung zu schärfen.

\section{Literatur}

[Abr ${ }^{+}$02] Abrahamsson, P.; Salo, O.; Ronkainen, J.; Warsta, J.: Agile software development methods. Review and analysis. VTT Electronic, Espoo, No 478, 2002.

[Acce04] Accenture: Was-will-der-Buerger.de - Online-Angebot und -Nachfrage im öffentlichen Sektor, o.O., Mai 2004.

[Acce02] Accenture: E-Government Leadership - High Performance, Maximum Value. The Government Executive Series, o.O., 2002.

[Ble ${ }^{+}$02a] Bleek, W.-G.; Jeenicke, M.; Klischewski, R.: Developing web-Based Applications through e-Prototyping. In: International Computer Software and Applications Conference, COMSAC 2002 (Oxford, England, August 26-29), Los Alamitos, CA, IEEE Computer Society, S. 609-614.

[Ble ${ }^{+}$02b] Bleek, W.-G.; Jeenicke, M.; Klischewski, R.: Framing Participatory Design Through e-Prototyping. In: Binder, Greogry, Wagner (Hg.): Proceedings of the Participatory Design Conference PDC 2002, CPSR, S. 300-305.

[BoKi97a] Bogumil, J.; Kißler, L.: Risiken und Chancen eines Neuen Steuerungsmodells für die lokale Demokratie. In: Bogumil, J., Kißler, L. (Hrsg.): Verwaltungsmodernisierung und lokale Demokratie. Baden-Baden: Nomos 1997, S. 9-19.

[BoKi97b] Bogumil, J.; Kißler, L.: Der Staat in Augenhöhe? Durch „Kundenorientierung“ zu einem neuen Verhältnis zwischen Staat und Bürger. In: Alternative Kommunalpolitik, Heft 1, 1997, S. 55-59.

[BoF103] Both, W.; Floren, A.: Modernisierung in Wirtschaft und Verwaltung - eine Umfrage bei Berliner Unternehmen. DE-CODA GmbH/Senatsverwaltung für Wirtschaft, Arbeit und Frauen, Berlin, 2003.

[BMI04] Bundesministerium des Innern (BMI): BundOnline - Basiskomponenten und Konpetenzzentren. Projektgruppe BundOnline, Berlin, Mai 2004.

[Floy84] Floyd, C.: A Systematic Look at Prototyping. In: Budde, R.; Kuhlenkamp, K.; Mathiassen, L.; Züllighoven, H. (Eds.): Approaches to Prototyping. Springer Verlag, Berlin, Heidelberg 1984, S. 1-18. 
[Flo $\left.{ }^{+} 89\right]$ Floyd, C.; Reisin, F.-M.; Schmidt, G.: STEPS to software development with users. In ESEC '89, Lecture Notes in Computer Science \#387, Berlin, Springer, 1989, S. 4864.

[Flo ${ }^{+}$04] Floyd, C.; Pape, B.; Bleek, W.-G.; Jackewitz, I.; Jeenicke, M.: Softwareentwicklung als Wissensprojekt. In: Pape, B.; Krause, D.; Oberquelle, H. (eds.): Wissensprojekte - Gemeinschaftliches Lernen aus didaktischer, softwaretechnischer und organisatorischer Sicht. Münster: Waxmann, 2004.

[Gare04] Gareis, K.: Towards User-centered E-Government - Understanding Demand for Online Public Services. Proceedings Telecities \& MUTEIS Conference „Urban Impacts of the Information Society“, The Hague, March 2004.

[Jee ${ }^{+}$03] Jeenicke, M.; Bleek, W.-G.; Klischewski, R.: Revealing Web User Requirements through e-Prototyping, In: Proceedings of the $15^{\text {th }}$ International Conference on Software Engineering and Knowledge Engineering (SEKE 03), San Francisco, USA, July 2003.

[KGSt93] Kommunale Gemeinschaftsstelle für Verwaltungsvereinfachung (KGSt): Das Neue Steuerungsmodell. Begründungen. Konturen. Umsetzungen, Ber. 5, Köln, 1993.

[KGSt94] Kommunale Gemeinschaftsstelle für Verwaltungsvereinfachung (KGSt): Das Neue Steuerungsmodell. Def. und Beschreibung von Produkten, Ber. 8, Köln, 1994.

[Len $\left.{ }^{+} 02\right]$ Lenk, K.; Traunmüller, R.; Wimmer, M. A.: The Significance of Law and Knowledge for Electronic Government. In: Grönlund, A. (ed.): Electronic Government - Design, Applications and Management. Idea Group, Hershey, London 2002 S. 61-77.

[Lenk99] Lenk, K.: Analyse des Verwaltungshandelns als Voraussetzung für die Ausschöpfung des Potentials der Informationstechnik. In: Lenk, K. Traunmüller, R. (Hg.): Öffentliche Verwaltung und Informationstechnik. Perspektiven einer radikalen Neugestaltung der öffentlichen Verwaltung mit Informationstechnik. Heidelberg, 1999, v. Decker, S. 53-70.

[Pap $\left.{ }^{+} 01\right]$ Pape, B.; Bleek, W.-G.; Jackewitz, I.; Janneck, M.: Software Requirements for Project-Based Learning - CommSy as an Exemplary Approach. Proceedings HICSS35, 2001.

[ShCh01] Sherrell, L. B.; Chen, L.-D.: The W Life Cycle Model and Associated Methodology for Corporate Web Site Development. Communications of the Association for Information Systems, 5 (7), April 2001.

[ScNa93] Schuler, D.; Namioka, A. (Hrsg.): Participatory design: principles and practices. Hillsdale, NJ: Lawrence Erlbaum Ltd., 1993.

[Somm01] Sommerville, I.: Software Engineering. Sixth Edition. Pearson Education Limited, England: Addison-Wesley, 2001.

[ $\mathrm{Kra}^{+}$97] Krabbel, A.; Wetzel, I.; Ratuski, S.: Anforderungsermittlung für Krankenhausinformationssysteme: Definition von Kernsystem und Ausbaustufen. In: Hasselbring, W. (Hrsg.): Erfolgsfaktoren Softwaretechnik für die Entwicklung von Krankenhausinformationssystemen. Krehl Verlag Münster, 1997, S. 1-8.

[Zwic04] Zwick, D.: Website bund.de wenig nutzerfreundlich. In: Wirtschaftswoche, wiwo.de, Abruf am 5.6.04. 